\title{
Natural Polymers in Tissue Engineering
}

\author{
Mashal Alenazi \\ University of Bridgeport, Biomedical Engineering Department, USA
}

\begin{abstract}
The application of freeze dried hydroxypropyl methylcellulose (HPMC) and methylcellulose (MC) as stabilizers in peanut butter were investigated. These cellulose derivatives were dispersed in water and subsequently freeze dried and chopped to produce a stabilizer that is able to absorb high amounts of oil. Without this templating approach, HPMC and MC had no effect on peanut butter stability and texture. An alternative spray drying approach was attempted. There are numerous techniques for microfabrication of patterned polymer surfaces and microchips for drug delivery. While silicon has been the choice material for much of the research done with MEMS, the methacrylates and acrylates provide a rapid and inexpensive base for future work. new method for determining Rigidity temperature applying cooling curve analysis. The determined values of Rigidity temperatures for three AlSi8Cu3 alloys with different contents of Strontium using thermal analysis technique have been compared with Rigidity temperatures obtained using mechanical technique (viscosity measurement).
\end{abstract}

Keywords: Natural, tissue, hydrolysate, polymers, engineering.

\section{INTRODUCTION}

Natural polymers are a class of polymeric materials that have natural (animal, plant, and algal) origins, consisting primarily of glycosidic linkages [1-6]. Natural polymeric raw materials hold a special importance to both industry and our daily life [7-15]. A global revival with regard to the utilization and interdisciplinary research of natural polymers has been spurred on due to the importance of renewable resources, the development of innovative products for science and technology through functional modifications, and an a bundant availability of natural polymers. The health effects of dietary fat consumption and the associated risk of cardiovascular disease (CVD) has been a controversial area of research over the past several decades (Ascherio et al., 1996; McGee, Reed, Yano, Kagan, \& Tillotson, 1984). The aim to reduce the consumption of saturated fat began with an epidemiological study, called the Seven Countries Study (Keys, 1970), which postulated a positive association between high intake of saturated fats and risk of CVD, and a negative association between high intake of monounsaturated fats and risk of CVD.

Since that time, many concerns regarding the validity of the Seven Countries Study have been raised (Reiser, 1973). Damrongsakkul et al. proposed the enzymatic hydrolysis of rawhide using two separate enzymes, papain and neutrase. Protein recovery rates increased sharply during the initial $10 \mathrm{~min}$ of enzymatic hydrolysis prior to a slowing down of the rate. The optimum working conditions of papain and neutrase for the highest protein recoveries were $708 \mathrm{C}$ at $\mathrm{pH} 6-7$, and $40-508 \mathrm{C}$ at $\mathrm{pH} 6-$ 7 , respectively. Due to the different hydrolysismechanisms of papain and neutrase, the obtained gelatins yielded different properties. Long peptide chains obtained from papain hydrolysis exhibited a solution sol-gel transition. On the other hand, neutrase severely attacked collagen molecules, resulting in short peptide chains of collagen hydrolysate that could not gel. Carbohydrates extracted/derived from natural resources have excellent biocompatibility and biodegradability, making them suitable for various medical, cosmetic, environmental, and food applications. The recent green chemistry has focused on natural hydrophilic polymers and developed variety of derived functional materials. The publications highlight new methodologies to prepare, characterize and apply materials for specific application needs and the current society's needs such as eco-friendly energy, global warming, and the interest in sustainability.

Although these new HT approaches may in part have been inspired by the similar application of HT concepts in the catalyst field, the development of these methods for polymer. esearch required new techniques be created specifically for the unique issues associated with polymers. These elegant advances are a challenge placed before the polymer community to create new more efficient analytical, synthetic, processing, and characterization methods useful for the study of other polymer problems. The goal of our research program is the development of a system of HT methods for rapid, detailed study of polymer nanocomposites. Whenever possible we attempt to keep the cost of the approach in mind and use standard commercially available equipment. An additional goal of our research focuses on the development of fundamental structure-property relations for polymer nanocomposites. Our primary interest is to develop an understanding of the governing, fundamental, mechanisms behind the enhanced mechanical properties and improved flammability properties of nanocomposites. Polymer nanocomposites are prepared by mixing a polymer (or monomer) with some dissimilar material, or additive, that has one or more dimensions on the nanometer scale. Over the last few decades, a wide variety of materials and synthesis approaches have been 
developed that allow molecular-level control over the coherency point solidification mode has been changed in design and structure of nanocomposite materials. favor of growth and coarsening of secondary dendrite Nanocomposites have been prepared by sol gel methods arms. The impingement of $\alpha$-aluminum crystals at dendrite [1], by in situ polymerization routes, and by using simple compounding methods. All of these approaches share a common theme; the intermingling, on the nanometer scale, of dissimilar materials for the purpose of creating new materials with properties not available from either of the component pure materials. ver the past two decades, a lot of coordination polymers [1](nonporous $\mathrm{CPs}$ and porous CPs also called metal-organic frameworks, MOFs) have been synthesized based on a variety of organic ligands and they exhibit the fascinating functional properties and potential applications in the fields such as luminescence [2e5], magnetism [6,7], gas storage [8e10], sensor, catalysis, ion exchange [17e19], and so on. Besides the properties, the coordination polymers also show us the charming architectural aesthetics in the microscopic universe, such as the interpenetration, interlocking, selfcatenation, helix, metal-carboxylate chainbased structures (also called rod-packing structures), zeolitic imidazolate frameworks featuring four-connected tetrahedral topologies, pillared layered structures and so on.

\section{DYNAMIC MECHANICAL ANALYSIS}

The chemicals used in this work are of analytical grade and were purchased from Jinan Henghua Sci. \& Tec. Co., Ltd and were used without further purification. The FT-IR spectra were measured with $\mathrm{KBr}$ pellets in the range of $4000 \mathrm{e} 400 \mathrm{~cm}$ 1on a PerkinElmer FT-IR Spectrometer. Elemental analyses for $\mathrm{C}, \mathrm{H}$ and $\mathrm{N}$ were performed on a PerkineElmer $240 \mathrm{C}$ analyzer. The thermogravimetric analyses (TGA) were performed in a SHIMADZU DTG60 simultaneous DTATG apparatus instrument, under dynamic $\mathrm{N}$ atmosphere $(20 \mathrm{~mL} / \mathrm{min})$ and heating rate of $102 \mathrm{C} / \mathrm{min}$ from room temperature. Powder X-ray diffraction investigation on polycrystalline samples were

carried out with a Bruker D8 advanced diffractometer equipped with a diffracted-beamed monochromator set for $\mathrm{Cu}$-Ka $(11 / 41.5418 \AA)$ radiation. The data were collected using a Ni-filtered Cu-target tube at room temperature in the $2 \mathrm{q}$ range from 5 at an angular rate of $0.1 \mathrm{~s} / \mathrm{step}$, with a scan step width of 0.02 . Photoluminescent spectra were measured using a PerkinElmer LS55 Fluorescence Spectrometer. The crystallization process of aluminum cast alloys begins with the development of primary $\alpha$ aluminum dendrite network. A dendrite is a characteristic tree like structure of crystal growing as molten metal freeze. Dendrites normally grow from a single nucleus both forwards (primary) and sideways (secondary) which may be only a few $\mu \mathrm{m}$ in diameter. During the early stages of the aluminum alloy solidification dendritic crystals are separate and move freely in the melt. However, as the melt cools, the dendrite tips of the growing crystals begin to impinge upon one another until a coherent dendritic network is formed. The temperature at which this occurs is called Dendrite Coherency Temperature (DCT) and is very coherency temperature lead to sharp decrease in the flow of the residual melt. Furthermore this temperature marked the moment when the "mass" feeding transferred to interdendritic feeding [5].

One of the basic ideas of modifying polymers with nanoplates of clays is to enhance the material's mechanical properties. DMA was frequently used in nanocomposites characterization since it allows the measurement of two different moduli of the nanocomposites, a storage modulus (E') which is related to the ability of the material to return or store mechanical energy and a loss modulus (E') which is related to the ability of the material to dissipate energy as a function of temperature. DMA data show significant improvements in the storage modulus over a wide temperature range of a number of polymer nanocomposites with MMT, such as PVDF, PP and PMMA. For all aluminum cast alloys, the transformation from liquid to solid state is accompanied by a decrease in volume in the ranges between $4 \%$ and $8 \%$, dependent on the type of alloy. In order to fulfill the volume deficit, the cast parts during solidification need to be fed with extra volume of liquid melt. The main intention in this case is to prevent shrinkage formation by maintaining a path for fluid flow from the higher heat mass and the pressure of the riser to the isolated liquid pool. Campbell [8] summarized the five characteristic feeding mechanisms that can occur during solidification of aluminum cast alloys. They are: liquid feeding, mass feeding, interdendritic feeding, burst feeding and solid feeding. Among these mechanisms liquid and mass feeding are relatively uncomplicated because of the low viscosity and wide active feeding path. After mass feeding resistance to melt flow increased considerably [4].

Polymer toxicity is something we'll have to investigate further, but during this study, we discovered that thioketal nanoparticles loaded with siRNA have a cell-toxicity profile similar to nanoparticles formulated from the FDAapproved material poly(lactic-co-glycolic acid)," said Murthy in the press release. In the future, thioketal nanoparticles may become a significant player in the treatment of numerous gastrointestinal diseases linked to intestinal inflammation, including gastrointestinal cancers, inflammatory bowel diseases and viral infections, according to Murthy.

The traditional definition of nanotechnology speaks of 'control of matter'. Many newly developed nanomedicines (e.g., targeted liposomes, polyplexes, nanotubes, modified/artificial viruses) can be designed to serve specific therapeutic purposes. Physicists, biologists, chemists, informatics experts, physicians, and pharmaceutical scientists all play a role in developing these 'smart' technologies for targeted delivery, for bioimportant feature of the solidification process. After imaging or for the development of new devices. In this 
context, as the first speaker (Daan Crommelin) proposed, the descriptor 'smart' technologies may be better than 'nanotechnologies', as in many cases the end product is in the micrometer range or larger and the feature that is of critical importance stands out not because of size but because of function.

\section{CONCLUSION}

The beneficial properties of natural hydrophilic polymers such as their biocompatibility, biodegradability, and nontoxicity have enabled them to be used in various applications having an enormous influence on one's daily life. The applications of chitosan, pectin, collagen, and gelatin have been rapidly updated with recent developments in various medical, environmental, and food technologies. I have seen that the structures of the current paths - fuzzy clusters - strongly depend on the degree of arrangement of the objects in the structure. The fuzzy clusters become more regular with increasing degree of arrangement of the objects. The same dependence can be found in the potential distributions of objects. The next consequence of it is increasing total electric current and the number of current paths in the structure. The differences between currents flowing in individual current paths become smaller. Freeze dried HPMC and MC can be used as alternative stabilizers to hydrogenated oils in peanut butter, and may be applied to other nut and seed butters. At addition levels between 1 and $2 \%$, the desirable oil stability and textural properties of commercial products (i.e., using hydrogenated oil stabilizers) can be achieved

\section{REFERENCES}

[1] Halake, K., Kim, H. J., Birajdar, M., Kim, B. S., Bae, H., Lee, C., Lee, J. (2016). Recently developed applications for natural hydrophilic polymers. Journal of Industrial and Engineering Chemistry, 40, 16-22. doi:

[2] Malujda, I., \& Wilczyński, D. (2016). Mechanical Properties Investigation of Natural Polymers. Procedia Engineering, 136, 263268. doi: http://dx.doi.org/10.1016/j.proeng.2016.01.208

[3] Peppas, N. A. (2004). Intelligent therapeutics: biomimetic systems and nanotechnology in drug delivery. Advanced Drug Delivery Reviews, 56(11), 1529-1531.

[4] A.K. Dillow, A. Lowman (Eds.), Biomimetic Materials and Design: Biointerfacial Strategies, Tissue Engineering and Targeted Drug Delivery, Dekker, New York, NY (2002)

[5] Tanti, R., Barbut, S., \& Marangoni, A. G. (2016). Oil stabilization of natural peanut butter using food grade polymers. Food $\begin{array}{llll}\text { Hydrocolloids, } & 61, & 399-408 . & \text { doi: }\end{array}$ http://dx.doi.org/10.1016/j.foodhyd.2016.05.034

[6] Crommelin, D. J. A., Park, K., \& Florence, A. (2010). Pharmaceutical nanotechnology: Unmet needs in drug delivery. Journal of Controlled Release, 141(3), 263-264.

[7] Tian, Z., Huang, J., Zhang, Z., Shao, G., Liu, A., \& Yuan, S. (2016). Organic-inorganic hybrid microporous polymers based on Octaphenylcyclotetrasiloxane: Synthesis, carbonization and adsorption for CO2. Microporous and Mesoporous Materials, 234, 130-136. doi: http://dx.doi.org/10.1016/j.micromeso.2016.06.038 